\title{
Conformational transformations induced by the charge-curvature interaction at finite temperature
}

Gaididei, Yuri Borisovich; Gorria, Carlos; Christiansen, Peter Leth; Sørensen, Mads Peter

Published in:

Physical Review E

Link to article, DOI:

10.1103/PhysRevE.78.051908

Publication date:

2008

Document Version

Publisher's PDF, also known as Version of record

Link back to DTU Orbit

Citation (APA):

Gaididei, Y. B., Gorria, C., Christiansen, P. L., \& Sørensen, M. P. (2008). Conformational transformations induced by the charge-curvature interaction at finite temperature. Physical Review E, 78(5), 051908. https://doi.org/10.1103/PhysRevE.78.051908

\section{General rights}

Copyright and moral rights for the publications made accessible in the public portal are retained by the authors and/or other copyright owners and it is a condition of accessing publications that users recognise and abide by the legal requirements associated with these rights.

- Users may download and print one copy of any publication from the public portal for the purpose of private study or research.

- You may not further distribute the material or use it for any profit-making activity or commercial gain

- You may freely distribute the URL identifying the publication in the public portal 


\title{
Conformational transformations induced by the charge-curvature interaction at finite temperature
}

\author{
Yu. B. Gaididei \\ Bogolyubov Institute for Theoretical Physics, Metrologichna street 14 B, 01413, Kiev, Ukraine \\ C. Gorria \\ Department of Applied Mathematics and Statistics, University of the Basque Country, E-48080 Bilbao, Spain \\ P. L. Christiansen \\ Informatics and Mathematical Modeling and Department of Physics, The Technical University of Denmark, DK-2800 Lyngby, Denmark \\ M. P. Sørensen \\ Department of Mathematics, The Technical University of Denmark, DK-2800 Lyngby, Denmark
}

(Received 30 April 2008; published 11 November 2008)

\begin{abstract}
The role of thermal fluctuations in the conformational dynamics of a single closed filament is studied. It is shown that, due to the interaction between charges and bending degrees of freedom, initially circular aggregates may undergo transformation to a polygonal shape. The transition occurs in the cases of hardening and softening charge-bending interaction. In the former case the charge and curvature are smoothly distributed along the chain, while in the latter spontaneous kink formation is initiated. The transition to a noncircular conformation is analogous to a phase transition of the second kind.
\end{abstract}

DOI: 10.1103/PhysRevE.78.051908

PACS number(s): 87.15.-v, 63.20.Pw, 63.20.Ry

\section{INTRODUCTION}

Conformational flexibility is a fundamental property of biological systems which determines their functioning [1-3]. Even modest conformational changes modify long-range electronic interactions in oligopeptides [4]; they may remove steric hindrances and open the pathways for molecular motions that are not available in rigid proteins [5]. The DNA conformation in the nucleosome core is crucial for gene replication, transcription, and recombination [6]. Recent DNA cyclization experiments [7-9] have shown the facile in vitro formation of DNA circles shorter than $30 \mathrm{~nm}$ (100 base pairs) which is even shorter than the commonly accepted persistence length $50 \mathrm{~nm}$ (150 base pairs). This means that the wormlike chain model does not work for such short DNA molecules, and to explain this phenomenon one should allow local softenings of DNA which facilitates disruptions (kinks) in the regular DNA structure [10-12]. According to [12] the kink formation is due to strong DNA bending while in [9] it is assumed that the softening originates from Watson-Crick base-pair breathing. An alternative approach which allows one to avoid kinking was proposed in Ref. [13], where a class of models with nonlinear DNA elasticity was introduced. It was shown in [13] that a "subelastic chain" model, in the frame of which the bending energy is proportional to the absolute value of curvature, can reproduce the main features of Cloutier and Widom's experiments [7].

Quite recently a simple, generic model for chargecurvature interactions on closed molecular aggregates was proposed [14]. It was shown that the presence of charge modifies (softens or hardens) the local chain stiffness. It was found that due to the interaction between charge carriers and the bending degrees of freedom the circular shape of the aggregate may become unstable and the aggregate takes the shape of an ellipse or, in general, of a polygon. It was shown also that when the charge-curvature interaction leads to soft- ening the local chain stiffness kinks spontaneously appear in the chain.

These results were obtained by using the mean-field approach where thermal fluctuations are ignored and strictly speaking, this approach is valid only for zero temperature. In the case of finite temperature, the interaction with environment and thermal fluctuations have to be considered.

The aim of this paper is to extend the results of Ref. [14] to the case of finite temperature. We study the chargeinduced conformational transformations of closed molecular aggregates in the presence of thermal fluctuations which we model in the frame of Langevin dynamics. The paper is organized as follows. In Sec. II we describe the model. In Sec. III we present an analytical approach to the problem. In Sec. IV we display the results of numerical simulations and compare with the analytical results. In Sec. V we discuss some concluding remarks.

\section{THE MODEL}

We consider a polymer chain consisting of $L$ units (for DNA each unit is a base pair) labeled by an index $l$, and located at the points $\vec{r}_{l}=\left(x_{l}, y_{l}\right), l=1, \ldots, L$. We are interested in the case when the chain is closed; therefore we impose the periodicity condition on the coordinates,

$$
\vec{r}_{l}=\vec{r}_{l+L} \text {. }
$$

We assume that there is a small number of mobile carriers (electrons, holes in the case of DNA, protons in the case of hydrogen-bonded systems) on the chain. The Hamiltonian of the system can be presented as the sum

$$
H_{\text {tot }}=H+H_{\text {stoch }} \text {. }
$$

The first term in this equation is the Hamiltonian of an isolated filament introduced in Ref. [14], 


$$
H=U_{b}+U_{s}+H_{\mathrm{el}}+H_{\mathrm{el}-\mathrm{conf}} .
$$

Here

$$
U_{b}=\frac{k}{2} \sum_{l} \frac{\kappa_{l}^{2}}{1-\kappa_{l}^{2} / \kappa_{\max }^{2}}
$$

is the bending energy of the chain, where

$$
\kappa_{l} \equiv\left|\vec{\tau}_{l}-\vec{\tau}_{l-1}\right|=2 \sin \frac{\alpha_{l}}{2}
$$

determines the curvature of the chain at the point $l$. Here

$$
\vec{\tau}_{l}=\frac{\vec{r}_{l+1}-\vec{r}_{l}}{\left|\vec{r}_{l+1}-\vec{r}_{l}\right|}
$$

is the tangent vector at the point $l$ of the chain, $\alpha_{l}$ is the angle between the tangent vectors $\vec{\tau}_{l}$ and $\vec{\tau}_{l-1}$, and $k$ is the elastic modulus of the bending rigidity (spring constant) of the chain. The term $\kappa_{l}^{2} / \kappa_{\max }^{2}$ in Eq. (4) gives the penalty for too large bending deformations. Here the parameter $\kappa_{\max }$ $=2 \sin \left(\alpha_{\max } / 2\right)$ is the maximum local curvature with $\alpha_{\max }$ being the maximum bending angle. The second term in Eq. (3),

$$
U_{s}=\frac{\sigma}{2} \sum_{l}\left(\left|\vec{r}_{l}-\vec{r}_{l+1}\right|-a\right)^{2},
$$

determines the stretching energy with $\sigma$ being an elastic modulus of the stretching rigidity of the chain and $a$ is the equilibrium distance between units (in what follows we assume $a=1)$. We take the simplest theoretical model for charge carriers, a nearest neighbor tight binding Hamiltonian, in the form

$$
H_{\mathrm{el}}=J \sum_{l}\left|\psi_{l}-\psi_{l+1}\right|^{2},
$$

where $\psi_{l}$ is the wave function of the carrier localized at $r_{l}$ and $J$ measures the carrier hopping between adjacent sites. The last term in Eq. (3) represents the charge-curvature interaction. In the small-curvature limit it has the form

$$
H_{\mathrm{el}-\mathrm{conf}}=-\frac{1}{2} \sum_{l} \chi\left|\psi_{l}\right|^{2}\left(\kappa_{l+1}^{2}+\kappa_{l-1}^{2}\right) ;
$$

here $\chi$ is the coupling constant. Combining Eqs. (4) and (9), we notice that the effective bending rigidity changes close to the points where the electron (hole) is localized. For positive values of the coupling constant $\chi$, there is a local softening of the chain, while for $\chi$ negative there is a local hardening of the chain.

The quantity

$$
\nu \equiv \frac{1}{L} \sum_{l}\left|\psi_{l}\right|^{2}
$$

gives the total density of charge carriers that can move along the chain and participate in the formation of the conformational state of the system. The second term in Eq. (2) describes the interaction of the filament with a fluctuating environment,

$$
H_{\text {stoch }}=\sum_{l} \vec{r}_{l} \cdot \vec{R}_{l}(t)
$$

where the stochastic forces $\vec{R}_{l}(t)=\left(X_{l}(t), Y_{l}(t)\right)$ are the Gaussian white noise,

$$
\begin{gathered}
\left\langle X_{l}(t)\right\rangle=\left\langle Y_{l}(t)\right\rangle=0, \\
\left\langle X_{l}(t) X_{l^{\prime}}\left(t^{\prime}\right)\right\rangle=\left\langle Y_{l}(t) Y_{l^{\prime}}\left(t^{\prime}\right)\right\rangle=2 D \delta_{l l^{\prime}} \delta\left(t-t^{\prime}\right), \\
\left\langle X_{l}(t) Y_{l^{\prime}}\left(t^{\prime}\right)\right\rangle=0,
\end{gathered}
$$

with $D$ the standard deviation.

To analyze the evolution of the shape of the filament, it is convenient to introduce the radius-of-gyration tensor $\mathbf{I}$ as in Refs. $[15,16]$. Its components are

$$
\begin{gathered}
\mathbf{I}_{x x}(t)=\frac{1}{L} \sum_{l}\left[x_{l}(t)-x^{c}(t)\right]^{2}, \\
\mathbf{I}_{y y}(t)=\frac{1}{L} \sum_{l}\left[y_{l}(t)-y^{c}(t)\right]^{2}, \\
\mathbf{I}_{x y}(t)=\frac{1}{L} \sum_{l}\left[x_{l}(t)-x^{c}(t)\right]\left[y_{l}(t)-y^{c}(t)\right],
\end{gathered}
$$

where

$$
\left(x^{c}(t), y^{c}(t)\right)=\frac{1}{L} \sum_{l}\left(x_{l}(t), y_{l}(t)\right)
$$

is the center-of-mass coordinate. The square roots of the two eigenvalues $R_{q}=\sqrt{\mathbf{I}_{q}}, q=1,2$, of the tensor $\mathbf{I}$ give the two principal radii of the system. They express the sizes of the filament along the major and minor axis. As is seen from Eqs. (13) the eigenvalues have the form

$$
\mathbf{I}_{1,2}=\frac{1}{2}\left[\left(\mathbf{I}_{x x}+\mathbf{I}_{y y}\right) \pm \sqrt{\left(\mathbf{I}_{x x}-\mathbf{I}_{y y}\right)^{2}+4 \mathbf{I}_{x y}^{2}}\right] .
$$

The indices 1 and 2 correspond to the + and - signs, respectively. To characterize the shape of the conformation it is convenient to introduce the quantity

$$
A=\mathbf{I}_{1}-\mathbf{I}_{2} \equiv \sqrt{\left(\mathbf{I}_{x x}-\mathbf{I}_{y y}\right)^{2}+4 \mathbf{I}_{x y}^{2}},
$$

defined as the "aspherity" [16]. It characterizes the shape's overall deviation from circular symmetry, which corresponds to $A=0$.

\section{ANALYTICAL APPROACH}

The aim of this section is to develop an analytical approach, which provides a better insight into the physical mechanism of conformational transformations induced by the charge-curvature interaction in the fluctuating media. We will assume that the characteristic size of the excitation is much larger than the lattice spacing and replace $\psi_{l}$ and $\vec{r}_{l}$ by the functions $\psi(s, t)$ and $\vec{r}(s, t)$, respectively. Here the arclength $s$ is the continuum analog of $l$. We assume that the 
chain is inextensible, and this assumption is expressed by the constraint

$$
\left|\partial_{s} \vec{r}\right|^{2}=1,
$$

which is automatically taken into account by choosing the parametrization

$$
\partial_{s} x(s)=\sin \theta(s), \quad \partial_{s} y(s)=\cos \theta(s),
$$

where the angle $\theta(s)$ satisfies the periodicity condition of Eq. (1),

$$
\begin{gathered}
\theta(s+L)=2 \pi+\theta(s) \\
\int_{0}^{L} \cos \theta(s) d s=\int_{0}^{L} \sin \theta(s) d s=0 .
\end{gathered}
$$

In the frame of the parametrization (18), the shape of the chain is determined by the equations

$$
x(s)=\int_{0}^{s} \sin \theta\left(s^{\prime}\right) d s^{\prime}, \quad y(s)=\int_{0}^{s} \cos \theta\left(s^{\prime}\right) d s^{\prime} .
$$

In the continuum limit the curvature (5) takes the form $\kappa(s)=\left|\partial_{s}^{2} \vec{r}(s)\right|$, which is given by

$$
\kappa(s)=\partial_{s} \theta .
$$

The continuum version of the total Hamiltonian of the system can be written as the sum

$$
H_{\text {tot }}=H+H_{\text {stoch }}(t) \text {, }
$$

where

$$
H=\int_{0}^{L}\left[J\left|\partial_{s} \psi\right|^{2}+\left(\frac{k}{2}-\chi\left|\psi^{2}\right|\right)\left(\partial_{s} \theta\right)^{2}\right] d s
$$

is the analog of the Hamiltonian (3) and

$$
H_{\text {stoch }}(t)=\int_{0}^{L}[X(s, t) x(s, t)+Y(s, t) y(s, t)] d s
$$

gives the interaction of the chain with the fluctuating environment (11) in the continuum limit. The stochastic forces $\vec{R}(s, t)=(X(s, t), Y(s, t))$ are the continuum version of the forces $X_{l}(t)$ and $Y_{l}(t)$. They obey the relations

$$
\begin{gathered}
\left\langle X(s, t) X\left(s^{\prime}, t^{\prime}\right)\right\rangle=\left\langle Y(s, t) Y\left(s^{\prime}, t^{\prime}\right)\right\rangle=2 D \delta\left(s-s^{\prime}\right) \delta\left(t-t^{\prime}\right), \\
\left\langle X(s, t) Y\left(s^{\prime}, t^{\prime}\right)\right\rangle=0 .
\end{gathered}
$$

We will restrict our analysis to the case when the filament shape only slightly deviates from the circle. Therefore in the derivation of the Hamiltonian (24) we can neglect the term $\kappa^{2} / \kappa_{\max }^{2}$ in the denominator of Eq. (4).

By using the Madelung transformation

$$
\psi(s, t)=\sqrt{\rho(s, t)} e^{i \phi(s, t)}
$$

where $\rho(s, t)$ is the charge density and $\phi(s, t)$ is the phase, the Hamiltonian (24) can be written as follows:

$$
H=\int_{0}^{L}\left[J\left(\frac{\left(\partial_{s} \rho\right)^{2}}{4 \rho}+\rho\left(\partial_{s} \phi\right)^{2}\right)+\left(\frac{k}{2}-\chi \rho\right)\left(\partial_{s} \theta\right)^{2}\right] d s .
$$

The dynamics of the system is governed by the Hamilton equations for the charge variables $\rho(s, t)$ and $\phi(s, t)$,

$$
\frac{\delta \mathcal{L}}{\delta \rho}=0 \quad \text { and } \frac{\delta \mathcal{L}}{\delta \phi}=0,
$$

where

$$
\mathcal{L}=-\int_{0}^{L} \rho \partial_{t} \phi d s-H
$$

is the Lagrangian of the system and $\delta / \delta(\cdot)$ is a variational derivative. By introducing the dissipation function

$$
\mathcal{F}=\eta \frac{1}{2} \int_{0}^{L}\left(\partial_{t} \vec{r}\right)^{2} d s
$$

the Langevin equation for the position $\vec{r}(s, t)$ can also be written in the variational form

$$
\frac{\delta \mathcal{F}}{\delta \partial_{t} \vec{r}(s, t)}=-\frac{\delta H_{\mathrm{tot}}}{\delta \vec{r}(s, t)} .
$$

Equations (29) and (32) should be considered with the periodicity condition (19) and the closure condition (20) for the position $\vec{r}(s, t)$. Now the periodicity conditions for the charge variables take the form

$$
\rho(s)=\rho(s+L), \quad \phi(s)=\phi(s+L),
$$

and the normalization condition in (10) becomes

$$
\frac{1}{L} \int_{0}^{L} \rho d s=\nu .
$$

To take into account the periodicity conditions (19) and (33), we can expand the curvature and the charge variables in the Fourier series

$$
\begin{gathered}
\partial_{s} \theta(s, t)=\frac{2 \pi}{L}\left[1+\sum_{j \geqslant 2} c_{j}(t) \cos \left(\frac{2 \pi j s}{L}\right)\right], \\
\rho(s, t)=\nu\left[1+\sum_{j \geqslant 2} \zeta_{j}(t) \cos \left(\frac{2 \pi j s}{L}\right)\right], \\
\phi(s, t)=\sum_{j \geqslant 2} \Phi_{j}(t) \cos \left(\frac{2 \pi j s}{L}\right) .
\end{gathered}
$$

Note that the first harmonic with $j=1$ does not contribute to the Fourier expansion (35) due to the closure condition (20). The coefficient $\nu$ in the expansion (36) takes into account the normalization condition (34). Different harmonics in the Fourier expansion (35) represent different types of shape deformation. For example, the term with $j=2$ determines an elliptic deformation, the term with $j=3$ represents a triagonal deformation, etc. (see [14] for more detail). For the sake of simplicity we consider only the elliptic deformation of the 
filament $(j=2)$ and restrict ourselves to the case when the deviations from the circular shape are small and the charge distribution along the chain is smooth: $\left|c_{2}\right|,\left|\rho_{2}\right|,\left|\phi_{2}\right|<1,\left|c_{j}\right|$, $\left|\rho_{j}\right|,\left|\phi_{j}\right| \ll 1$ for $j \geqslant 3$. Thus the expansions (35)-(37) reduce to

$$
\begin{gathered}
\partial_{s} \theta(s, t)=\frac{2 \pi}{L}\left[1+c(t) \cos \left(\frac{4 \pi s}{L}\right)\right], \\
\rho(s, t)=\nu\left[1+\zeta(t) \cos \left(\frac{4 \pi s}{L}\right)\right], \\
\phi(s, t)=\Phi(t) \cos \left(\frac{4 \pi s}{L}\right),
\end{gathered}
$$

where we omitted the subscript in the notations for the Fourier harmonics.

Inserting Eqs. (38)-(40) into Eqs. (25), (28), (30), and (31), we get

$$
\mathcal{L}_{\text {eff }}=-\frac{L}{2} \nu \zeta \frac{d \Phi}{d t}-\mathcal{H}
$$

Here

$$
\mathcal{H}=\frac{\pi^{2}}{L}\left(-4 J \nu \sqrt{1-\zeta^{2}}+8 J \nu \Phi^{2}+k_{e} c^{2}-4 \chi \nu \zeta c\right)
$$

is the effective Hamiltonian with some irrelevant constants being omitted. In Eq. (42) $k_{e}=k-2 \chi \nu$ is an effective bending rigidity of the filament for the case when the charge is unformly distributed along the chain,

$$
\begin{aligned}
\mathcal{H}_{\text {stoch }}(t)= & \frac{L^{2}}{4 \pi^{2}} \int_{0}^{2 \pi}\left[X\left(\frac{L s}{2 \pi}, t\right) \int_{0}^{s} \cos \left(s^{\prime}-\frac{c}{2} \sin \left(2 s^{\prime}\right)\right) d s^{\prime}\right. \\
& \left.+Y\left(\frac{L s}{2 \pi}, t\right) \int_{0}^{s} \cos \left(s^{\prime}+\frac{c}{2} \sin \left(2 s^{\prime}\right)\right) d s^{\prime}\right] d s
\end{aligned}
$$

is the effective interaction with stochastic forces, and

$$
\mathcal{F}=\frac{1}{2} b(c)\left(\frac{d c}{d t}\right)^{2}
$$

is the effective dissipative function. The damping coefficient $b(c)$ has the form

$$
b(c)=\frac{1}{4} \eta \frac{L^{3}}{(2 \pi)^{3}} \int_{0}^{2 \pi}\left[\alpha^{2}(c, s)+\alpha^{2}(-c, s)\right] d s,
$$

where the notation

$$
\alpha(c, s)=\int_{0}^{s} \sin \left(2 s^{\prime}\right) \sin \left(s^{\prime}+\frac{c}{2} \sin \left(2 s^{\prime}\right)\right) d s^{\prime}
$$

is introduced. Note also that in the derivation of Eq. (43) we took into account the periodicity in the stochastic terms, $X(s+L, t)=X(s, t)$ and $Y(s+L, t)=Y(s, t)$.

Equations of motion for the quantitites $\Phi, \zeta$, and $c$ follow from Eqs. (28), (29), and (41)-(44) and they have the form

$$
\frac{d \Phi}{d t}=-\frac{8 \pi^{2}}{L^{2}}\left(J \frac{\zeta}{\sqrt{1-\zeta^{2}}}-\chi c\right),
$$

$$
\begin{gathered}
\frac{d \zeta}{d t}=\frac{32 \pi^{2}}{L^{2}} J \Phi, \\
\frac{d c}{d t}=-\frac{2 \pi^{2}}{b(c) L}\left(k_{e} c-2 \chi \nu \zeta\right)-f(c, t),
\end{gathered}
$$

where

$$
\begin{aligned}
f(c, t)= & \frac{L^{2}}{4 b(c) \pi^{2}} \int_{0}^{2 \pi}\left[X\left(\frac{L s}{2 \pi}, t\right) \alpha(-c, s)\right. \\
& \left.-Y\left(\frac{L s}{2 \pi}, t\right) \alpha(c, s)\right] d s
\end{aligned}
$$

is an effective stochastic force.

Note that, in terms of the ansatz (38), the aspherity $A$, which is defined by Eq. (16), can be written approximately as

$$
A=\frac{L^{2}}{8 \pi^{2}} c .
$$

Let us analyze the cases of zero temperature and finite temperature separately.

\section{A. Deterministic behavior: Zero-temperature limit}

In the no-noise case the dynamics of the system is described by Eqs. (47)-(49) with $X=Y=0$. The system under consideration is characterized by the control parameter

$$
\xi=\frac{\Delta_{\text {def }}}{\Delta_{\text {disp }}},
$$

which is the ratio of the deformation energy (i.e., the energy shift due to the charge-bending interaction)

$$
\Delta_{\mathrm{def}}=\left(\frac{2 \pi}{L}\right)^{2} \nu^{2} \frac{\chi^{2}}{k_{e}}
$$

with respect to the dispersion energy

$$
\Delta_{\text {disp }}=\frac{1}{2}\left(\frac{2 \pi}{L}\right)^{2} \nu J .
$$

A simple analysis shows that, when the charge-curvature coupling is weak such that the control parameter $\xi<1$, these equations have a unique stationary point $\Phi=0, \zeta=0$, and $c$ $=0$. This state corresponds to a uniformly distributed charge along the circular filament.

When $\xi>1$ there are two equivalent stationary states

$$
\Phi=0, \quad \zeta_{ \pm}= \pm \sqrt{1-\frac{1}{\xi^{2}}}, \quad c_{ \pm}=\frac{2 \chi \nu}{k_{e}} \zeta_{ \pm},
$$

which represent an elliptically deformed filament with a spatially nonuniformly distributed charge. The two solutions $c_{+}$ correspond to two mutually orthogonal directions in which the filament may be elongated. Note that in the case of softening charge-curvature interaction $(\chi>0)$ the maxima of the curvature and the charge density coincide, while in filaments with hardening charge-curvature interaction $(\chi<0)$ the cur- 
vature of the filament is minimal (the filament is locally more flat) in the places where the charge density is maximal.

\section{B. Charge-charge correlation effects}

The aim of this section is to clarify the role of interaction between charge carriers in the formation of polygonally shaped aggregates in the zero-temperature limit. In describing the charge-charge repulsion effects, we will use an onsite interaction in the form

$$
H_{\mathrm{el}-\mathrm{el}}=\frac{1}{2} V \sum_{n}\left|\psi_{n}\right|^{4},
$$

which in the continuum limit in terms of the charge variables (27) reads

$$
H_{\mathrm{el}-\mathrm{el}}=\frac{1}{2} V \int_{0}^{L} \rho^{2} d s .
$$

The parameter $V$ in Eqs. (56) and (57) characterizes the strength of the interaction. Thus the Hamiltonian of the system with account of charge-charge interaction effects has the form

$$
H_{\mathrm{cc}}=H+\frac{1}{2} V \int_{0}^{L} \rho^{2} d s,
$$

where the Hamiltonian $H$ is given by Eq. (28). Inserting in Eq. (58) the ansatz (38), we get

$$
\begin{aligned}
\mathcal{H}_{\mathrm{cc}} & =\frac{\pi^{2}}{L} \\
& \times\left(-4 J \nu \sqrt{1-\zeta^{2}}+\frac{L^{2}}{4 \pi^{2}} V \nu^{2} \zeta^{2}+8 J \nu \Phi^{2}+k_{e} c^{2}-4 \chi \nu \zeta c\right) .
\end{aligned}
$$

An inspection of the function (59) shows that in the zerotemperature limit the spatially uniform charge distribution along the circular filament becomes unstable for the control parameter (52) satisfying the inequality

$$
\xi>1+\frac{L^{2}}{8 \pi^{2}} \nu \frac{V}{J} .
$$

Thus the elliptic shape more easily arises in short filaments with strong charge-bending interaction $\chi$ and relatively weak interaction between charge carriers $V$.

In what follows we will assume that the charge-bending interaction is strong, $\Delta_{\text {def }} \gg \nu^{2} V / 4$, and for the sake of simplicity will neglect the interaction between charges.

\section{Stochastic behavior: Finite temperature}

We will study the role of thermal fluctuations by using the formalism of the Fokker-Planck equation. To this end we introduce the probability distribution density

$$
P(c, \zeta, \Phi ; t)=\langle\delta(c-c(t)) \delta(\zeta-\zeta(t)) \delta(\Phi-\Phi(t))\rangle .
$$

As seen from Eqs. (26) and (50) the stochastic forces $f(c, t)$ represent Gaussian white noise with the mean value

$$
\langle f(c, t)\rangle=0
$$

and the two-time covariance given by

$$
\left\langle f(c, t) f\left(c^{\prime}, t^{\prime}\right)\right\rangle=2 F\left(c, c^{\prime}\right) \delta\left(t-t^{\prime}\right),
$$

where

$$
\begin{aligned}
F\left(c, c^{\prime}\right)= & \frac{D}{b(c) b\left(c^{\prime}\right)} \frac{L^{3}}{8 \pi^{3}} \int_{0}^{2 \pi}\left[\alpha(-c, s) \alpha\left(-c^{\prime}, s\right)\right. \\
& \left.+\alpha(c, s) \alpha\left(c^{\prime}, s\right)\right] d s .
\end{aligned}
$$

It is straightforward to obtain (see, e.g., [17]) that the Fokker-Planck equation which describes the time evolution of the probability distribution (61) of the set of Langevin equations (47)-(49) in the Stratonovich sense has the form

$$
\begin{aligned}
\partial_{t} P= & -\partial_{\zeta}\left(P \partial_{\Phi} \mathcal{H}\right)+\partial_{\Phi}\left(P \partial_{\zeta} \mathcal{H}\right)+\partial_{c}\left(\frac{P}{b(c)} \partial_{c} \mathcal{H}\right) \\
& -\partial_{c}\left\{\left.P\left[\partial_{c} F\left(c, c^{\prime}\right)\right]\right|_{c^{\prime}=c}\right\}+\partial_{c}^{2}[P F(c, c)],
\end{aligned}
$$

where the Hamiltonian $\mathcal{H}$ is given by Eq. (42). Inserting into Eq. (65) the relations

$$
F(c, c)=\frac{T}{b(c)},\left.\quad \partial_{c} F\left(c, c^{\prime}\right)\right|_{c^{\prime}=c}=\frac{T}{2} \frac{d}{d c}\left(\frac{1}{b(c)}\right),
$$

which follow from Eqs. (45), (46), and (64), we obtain the equation for the probability distribution $P(c, \zeta, \Phi ; t)$ in the form

$$
\begin{aligned}
\partial_{t} P= & -\partial_{\zeta}\left(P \partial_{\Phi} \mathcal{H}\right)+\partial_{\Phi}\left(P \partial_{\zeta} \mathcal{H}\right)+\partial_{c}\left(\frac{P}{b(c)} \partial_{c} \mathcal{H}\right) \\
& -\frac{T}{2} \partial_{c}\left[P \frac{d}{d c}\left(\frac{1}{b(c)}\right)\right]+T \partial_{c}^{2}\left(\frac{1}{b(c)} P\right) .
\end{aligned}
$$

It is interesting to note that if the stochastic force $f(c, t)$ in the Langevin equations (47)-(49) is replaced by

$$
f_{\text {mod }}=\frac{1}{\sqrt{b(c)}} \xi(t),
$$

where $\xi(t)$ is a white noise with

$$
\langle\xi(t)\rangle=0, \quad\left\langle\xi(t) \xi\left(t^{\prime}\right)\right\rangle=2 D \delta\left(t-t^{\prime}\right),
$$

we obtain the same Fokker-Planck relation for this new set of Langevin equations. This means that the stochastic equations (47)-(49) with $f(c, t)$ from Eqs (26) and (50) or from Eqs. (68) and (69) are equivalent.

The stationary probability distribution [i.e., the solution of the Fokker-Planck equation (67) for $t \rightarrow \infty$ ] is given by

$$
P_{\mathrm{st}}=C \sqrt{b(c)} e^{-\mathcal{H} / T}
$$

where $C$ is a normalization constannt.

Assuming that $c<1$, one can expand the function $a(c, s)$ from Eq. (46) into a Taylor series and, keeping only leading terms, one can obtain that

$$
\ln b(c) \approx \text { const }+0.04 c^{2} \text {. }
$$

Combining Eqs. (42), (70), and (71) we obtain that the effective bending rigidity [i.e., the coefficient in front of $c^{2}$ in 


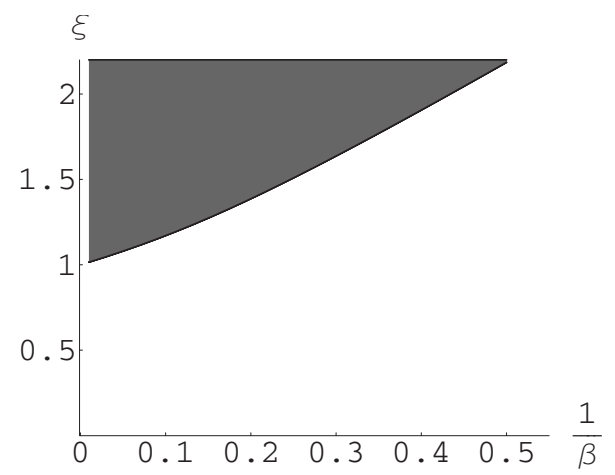

FIG. 1. Probability distribution phase diagram. In the shaded area the probability distribution is bimodal and in the unshaded area it is unimodal.

the expression $\mathcal{H}+\frac{1}{2} \ln b(c)$ ] becomes temperature dependent and takes the form $k_{e}-0.002 L T$. We will assume that the persistence length $l_{p}=k_{e} / T$ satisfies the inequality $l_{p}$ $\gg 0.002 L$, and in what follows we will neglect the temperature dependence of the effective bending rigidity.

By integrating the stationary probability distribution (70) over the charge variables $\Phi$ and $\zeta$ [note that $\zeta \in(-1,1)$ because by definition the charge density $\rho(s, t)>0]$ we obtain a reduced distribution

$$
\begin{aligned}
\mathcal{P}(c)= & \frac{1}{\mathcal{N}} \int_{0}^{\pi / 2} d \theta \sin \theta e^{\beta \sin \theta} \cosh \left(\beta \frac{\chi}{J} c \cos \theta\right) \\
& \times \exp \left[-\frac{\beta}{2 \xi}\left(\frac{\chi}{J}\right)^{2} c^{2}\right],
\end{aligned}
$$

where

$$
\begin{aligned}
\mathcal{N}= & \int_{-\infty}^{\infty} d c \int_{0}^{\pi / 2} d \theta \sin \theta e^{\beta \sin \theta} \cosh \left(\beta \frac{\chi}{J} c \cos \theta\right) \\
& \times \exp \left[-\frac{\beta}{2 \xi}\left(\frac{\chi}{J}\right)^{2} c^{2}\right]
\end{aligned}
$$

is the normalization constant. In Eqs. (72) and (73) $\beta$ $=4 \pi^{2} J \nu /(L T)$ is a dimensionless inverse temperature. The function $\mathcal{P}(c)$ gives the probability of finding the curvature $c$ in the interval $(c, c+d c)$ irrespective of the magnitude of the charge variables.

There are two areas in the parameter space $(\beta, \xi)$ where the probability density (72) as a function of the curvature parameter $c$ behaves qualitatively differently, as is shown in Fig. 1. These two areas are separated by the curve

$$
\xi=\frac{\beta}{\pi} \frac{2+\pi\left[I_{1}(\beta)+\mathbf{L}_{1}(\beta)\right]}{\beta\left[I_{0}(\beta)+\mathbf{L}_{0}(\beta)\right]-2\left[I_{1}(\beta)+\mathbf{L}_{1}(\beta)\right]},
$$

where $I_{n}(\beta)$ is the Bessel function of imaginary argument and $\mathbf{L}_{n}(\beta)$ is the Struve function [18]. Below this curve (i.e., in the unshaded area of the phase diagram presented in Fig. 1) the distribution is single modal. The probability density (72) in this case has a maximum at $c=0$ (see Fig. 2 when $T=0.3$ ). This means that the most probable conformation state of the filament is a circle. Above the curve (74) (i.e., in

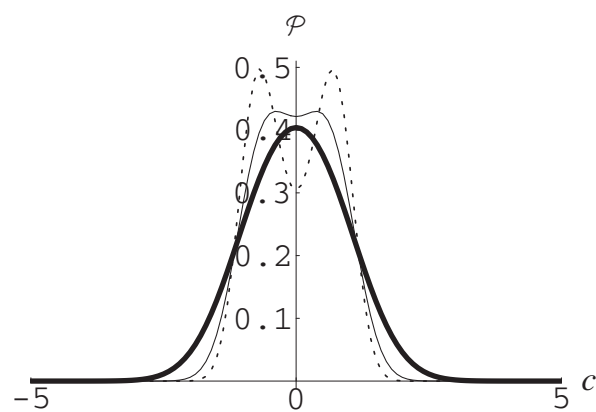

FIG. 2. Probability density distribution of $c$ variable for $J=k_{e}$ $=1, \nu=0.25, \chi=2, \sigma=10^{5}$, and three different values of the temperature: $T=0.05$ (dashed curve), 0.1 (thin curve), and 0.3 (thick curve)

the shaded area of Fig. 1) the probability distribution is bimodal. The function (72) has two equivalent maxima $\pm c_{m}$ (see Fig. 2 when $T=0.05$ and 0.1 ). In this case the most probable state is an elliptically deformed filament. The two maxima correspond to two mutually orthogonal directions of elongation.

It is straightforward to obtain that in the vicinity of the curve given by Eq. (74) the most probable value of the chain curvature is determined by the expression $c_{m}=\gamma(\xi) \sqrt{T_{c}-T}$ where $T_{c}(\xi)$ is a critical temperature [i.e., the solution of Eq. (74)] and $\gamma(\xi)$ is some coefficient. Thus, in the framework of our ansatz (38) the transition of the filament in the fluctuating environment to a noncircular conformation may be considered as a noise-induced phase transition of the second kind.

By using the probability distribution (72) and the expression (51) for the filament aspherity, one can calculate the equilibrium value of the normalized aspherity,

$$
\frac{\langle A\rangle}{\left\langle A_{0}\right\rangle}=\frac{M_{1}(T)}{M_{1}(0)},
$$

and the equilibrium value of the relative standard deviation of the aspherity,

$$
\frac{\sqrt{\left\langle(\Delta A)^{2}\right\rangle}}{\langle A\rangle}=\sqrt{\frac{M_{2}(T)}{M_{1}^{2}(T)}-1},
$$

where $M_{n}(T)$ are the moments defined as

$$
M_{n}(T)=\int_{-1}^{1} \mathcal{P}(c)|c|^{n} d c, \quad n=1,2, \ldots .
$$

\section{NUMERICAL STUDIES}

The dynamics of the filament is described by the Schrödinger equations

$$
i \frac{d}{d t} \psi_{l}=-\frac{\partial H}{\partial \psi_{l}^{*}}
$$

and the Langevin equations 


$$
\eta \frac{d}{d t} \vec{r}_{l}=-\frac{\partial H}{\partial \vec{r}_{l}}+\vec{R}_{l}(t)
$$

with the Hamiltonian $H$ being defined by Eq. (3). Thus the conformational dynamics is considered in an overdamped regime with $\eta$ being the friction coefficient. In accordance with the fluctuation-dissipation theorem, the standard deviation $D$ is proportional to the temperature $T$; thus $D=\eta T$.

The set of stochastic differential equations (78) and (79) is solved numerically by the use of an implicit Euler method, with $\alpha=0.5$ as implicitness parameter, which is the trapezoidal rule. The integration of the stochastic term is done by using the strong Taylor scheme of first order described in Ref. [19]. The time step chosen for running the simulations was $\Delta t=10^{-2}$. To verify the precision of the results we compared with data obtained for different time steps. The value of the position and charge of the $l$ th particle at time $t_{n}=n \Delta t$ is denoted $Z_{n}^{l}=\left(x_{l}, y_{l}, \phi_{l}\right)_{n}, F\left(\vec{Z}_{n}\right)$ denotes the deterministic part of Eqs. (78) and (79), and $W_{n}=\left(X_{l}\left(t_{n}\right), Y_{l}\left(t_{n}\right), 0\right)$ is the corresponding component of the white noise. Thus the numerical scheme becomes

$$
Z_{n+1}^{l}=Z_{n}^{l}+\left[\alpha F\left(\vec{Z}_{n+1}\right)+(1-\alpha) F\left(\vec{Z}_{n}\right)\right] \Delta t+W_{n} \sqrt{\Delta t} .
$$

The above system of nonlinear equations is implicit and it is solved by a hybrid method provided by the MINPACK FORTRAN library and the random numbers present in the white noise are generated by the RANLIB library, both accesible from the netlib repository in Ref [20].

In our paper we were mostly concerned with the role of thermal fluctuations in the process of the shape transformations. Therefore in our simulations we have chosen a set of parameters for which in the zero-temperature limit the most energetically favorable state is an elliptically deformed filament. Without loss of generality, in this section we show the results of the numerical simulations produced for $L$ $=36$ units and charge density $\nu=0.5$. A system of this size provides a clear visualization of the properties of the model and does not demand too much computational time. Systems of the same size but with smaller values of the charge density (but still inside the area where the elliptic state is stable) in the presence of thermal fluctuations require more time to reach an equilibrium state. As initial condition for the electric charge density $\psi_{l}$ we use the same magnitude at all points, corresponding to an equally distributed charge density. Initially, all the lattice points were symmetrically distributed along the circle of an appropriate radius. In what follows we have chosen the damping coefficient $\eta$ and the bending rigidity $k$ equal to unity. Moderate changes in these parameters do not modify significantly the dynamics of the system. To avoid big stretching of the nearest bonds of the chain we fix the parameter $\sigma=10^{5}$. We considered the cases of both hardening and softening electron-curvature interaction.
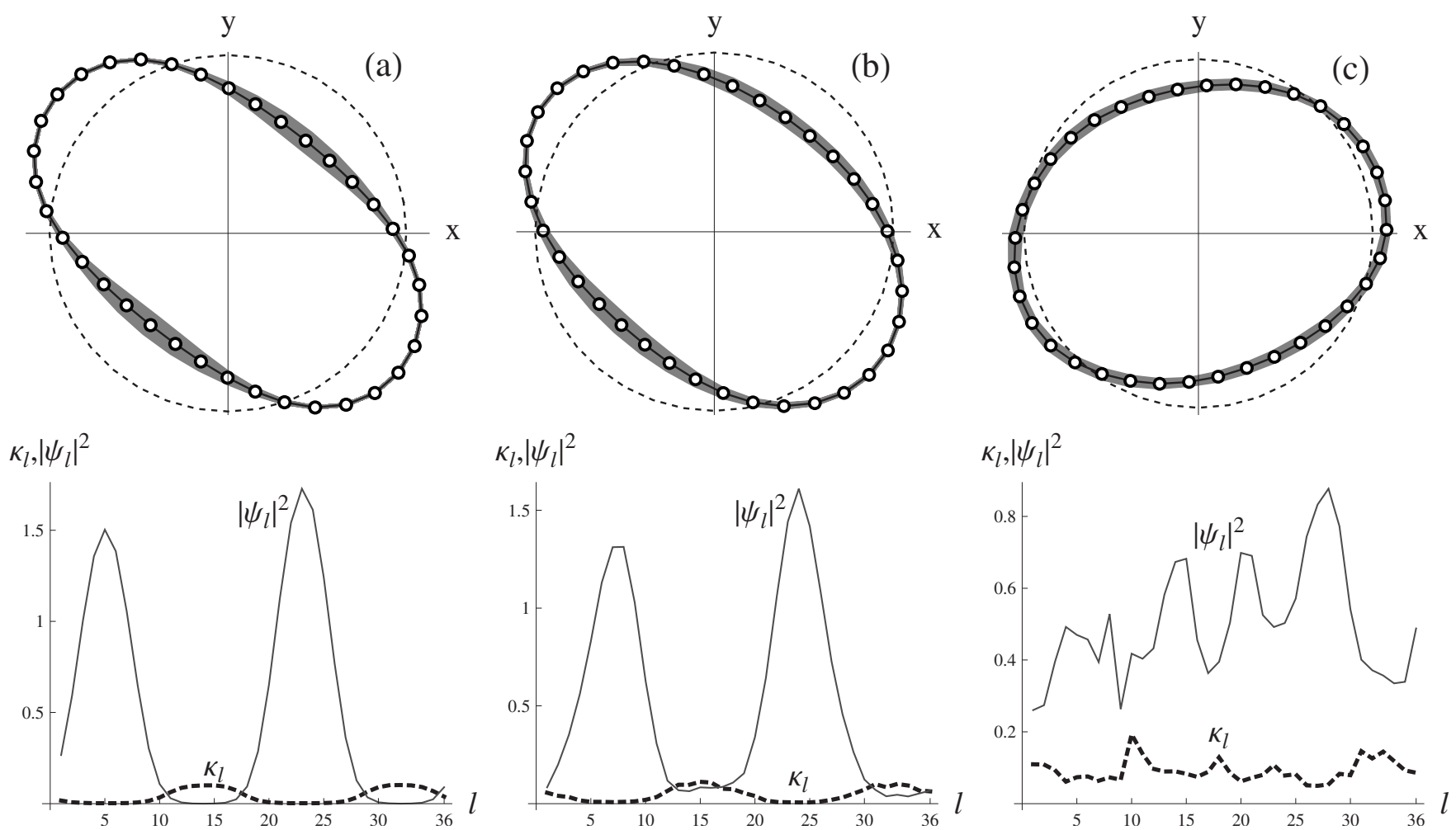

FIG. 3. Top panel: Equilibrium shape of the chain (solid line) against the initial circular shape (dashed line). Bottom panel Charge distribution $|\psi|^{2}$ (solid line) and curvature $\kappa$ (dashed line) for different noise intensity $D=0.01$ (a), 0.2 (b), and 0.4 (c) in the case of hardening, $\nu=0.5, \chi=-4, \sigma=10^{5}$, and $J=0.25$ at time $t=4000$. The gray shadow represents the local charge density in the chain. 

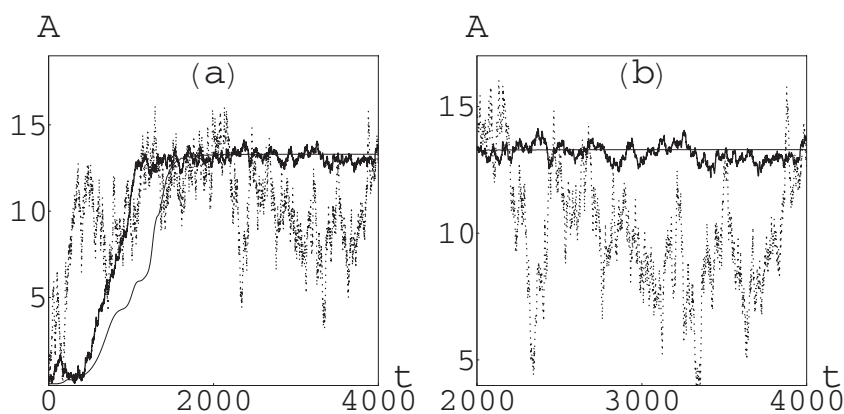

FIG. 4. Aspherity versus time for different noise intensity, $D$ $=0$ (thin line), 0.05 (thick line), and 0.25 (dotted line). The parameters used are $\nu=0.5, \chi=-4, \sigma=10^{5}$, and $J=0.25$. (a) Full time simulation $0 \leqslant t \leqslant 4000$; (b) detailed behavior after transient time $2000 \leqslant t \leqslant 4000$

\section{A. The case of hardening charge-curvature interaction}

Typical final shapes of the filament for three different values of the noise intensity $D=0.01,0.1$, and 0.2 are shown in Fig. 3 where the averaged aspherity decreases as the noise increases. Figure 4 shows the time evolution of the aspherity $A$ for three different values of the noise $D$. The left panel shows the overall behavior of this quantity (including transient processes) while the right panel presents its steady state evolution. As is seen from Fig. 4 the thermal fluctuations excite the dynamics of the system and facilitate the transition to an anisotropic state. Clearly the transient period shortens when the temperature increases.

It is remarkable that in the case of weak noise, $D=0.01$, in the aspherity time evolution there exists a small plateau for $600<t<1000$ (see Fig. 4). This plateau corresponds to an intermediate conformation of the filament when it takes a triangular shape (see Fig. 5).

The mean value of the saturation aspherity $\langle A\rangle$ after transient time $t_{1}$ which we define as

$$
\langle A\rangle=\frac{1}{t_{2}-t_{1}} \int_{t_{1}}^{t_{2}} A(t) d t
$$

decreases as the temperature increases (see Fig. 6) and the filament takes on a less anisotropic shape, while the relative standard deviation

$$
\frac{\sqrt{\left\langle(\Delta A)^{2}\right\rangle}}{\langle A\rangle}
$$

is an increasing function of temperature. Here the aspherity variance is given by the expression

$$
\left\langle(\Delta A)^{2}\right\rangle=\frac{1}{t_{2}-t_{1}} \int_{t_{1}}^{t_{2}}(A(t)-\langle A\rangle)^{2} d t .
$$

The shape of the aggregate is well defined when $\sqrt{\left\langle(\Delta A)^{2}\right\rangle} /\langle A\rangle \ll 1$. There is a critical value of the noise intensity $D_{\text {cr }}$ when the relative standard deviation reaches the value $1 / 2$. Then for $D>D_{\text {cr }}$ the shape fluctuations are so strong that they become chaotic. For $\nu=0.5$ this value is approximately $D_{\mathrm{cr}} \approx 0.4$.
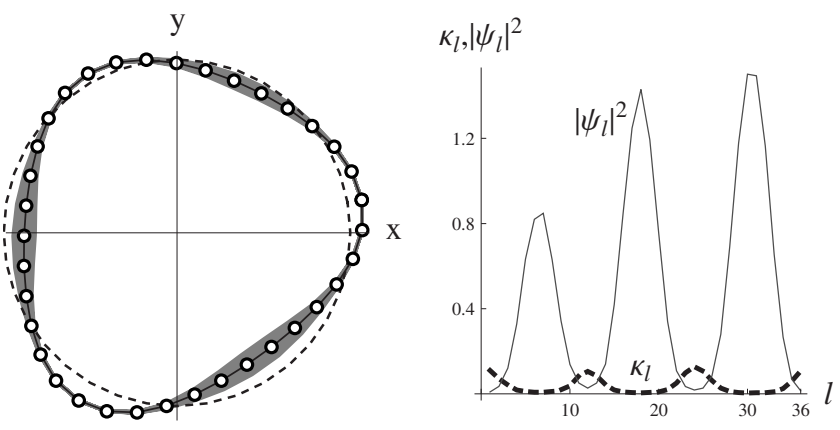

FIG. 5. Intermediate shape of the filament at time $t=500$ (left panel) and the corresponding charge distribution $|\psi|^{2}$ (solid line) and curvature $\kappa$ (dashed line) along the chain (right panel) for $D$ $=0.01$. The parameters used are the same as in Fig. 3 .

Thus one can conclude that in the case of hardening charge-bending interaction the mean-field approach introduced in [14] works well in the weak-noise limit.

(i) The ellipselike conformation is the equilibrium state of the shape evolution.

(ii) In the equilibrium state the charge distribution is nonuniform along the chain and the charge is concentrated in the places where the filament is more flat.

(iii) The triangular conformation of the filament exists but it is a metastable state.

Strong noise (i.e., high temperature) qualitatively changes the conformational dynamics keeping the shape of the filament almost circular, while in the zero-temperature limit it takes on an elliptic shape. The corresponding quantities are shown in Fig. 6. Comparing these relations with the numerical data, also presented in this figure, one can conclude that there is a qualitative agreement between the analytical results obtained in the framework of the simple ansatz (38) and the results of numerical simulations.

\section{B. The case of softening charge-curvature interaction}

By carrying out the simulations described for the hardening charge-bending interaction $(\chi<0)$ we took into account

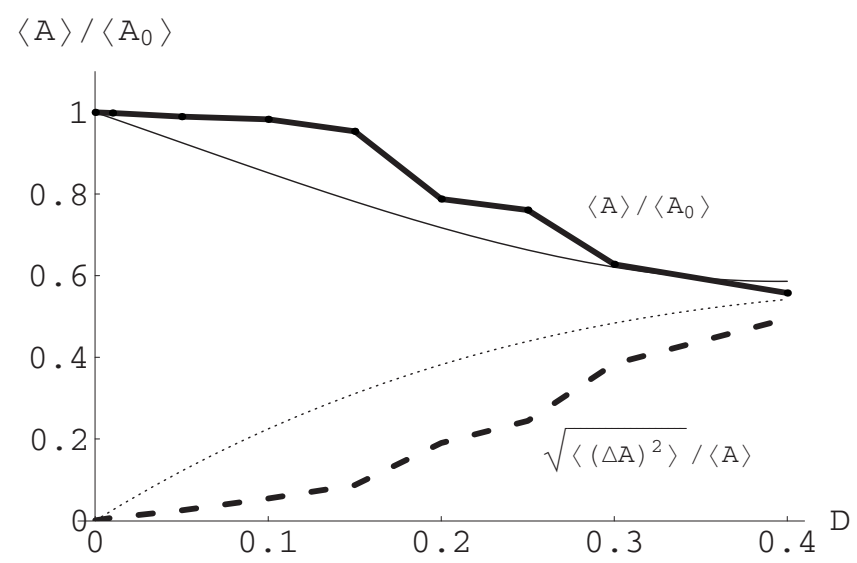

FIG. 6. Normalized mean value of the aspherity $\langle A\rangle /\left\langle A_{0}\right\rangle$ (thick solid line) and its relative standard deviation $\sqrt{\left\langle(\Delta A)^{2}\right\rangle} /\langle A\rangle$ (thick dashed line) versus noise from the simulations and as stationary solution of the Fokker-Planck equation (thin solid and dotted line, respectively) in the case of hardening, $\nu=0.5, \chi=-4, \sigma=10^{5}$, and $J=0.25$. 

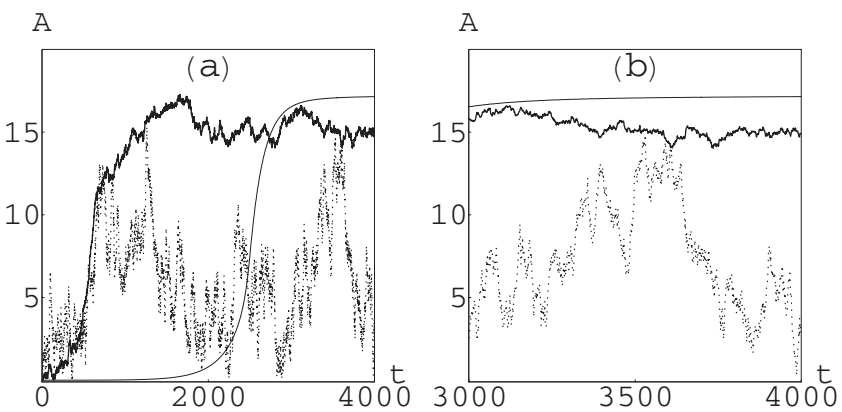

FIG. 7. Same as Fig. 4 for the case of softening, $\nu=0.29, \chi=2$, $\sigma=10^{5}$, and $J=0.22$. (a) Full time simulation $0 \leqslant t \leqslant 4000$; (b) detailed behavior after transient time $3000 \leqslant t \leqslant 4000$

that the presence of charge increases the bending rigidity locally and the large bending deformations do not occur. Therefore there was no necessity to introduce any bending energy penalty and in Eq. (4) we put $\kappa_{\max } \rightarrow \infty$. However, the filaments with softening charge-bending interaction are much more flexible and large deformations with the bending angle $\alpha_{l}>\pi / 2$ appear rather easily. To avoid excessive bending we carried out our numerical simulations under the assumption that the maximum possible bending angle $\alpha_{\max }$ is equal to $\pi / 2$. This means that we let $\kappa_{\max }=\sqrt{2}$ in Eq. (4).

Comparing the time evolution and the mean value of the aspherity in the case of softening charge-bending interaction presented in Fig. 7 and 8 with the case of hardening chargebending interaction (Figs. 4 and 6), we see that they are qualitatively similar. However, the equilibrium shapes of the filaments (Fig. 9) and the charge distribution (Fig. 10) after transient time $t=4000$ differ drastically. The softening charge-bending interaction initiates kink formation while smooth shapes of filaments are characteristic for the case of hardening charge-bending interaction. Almost all the charge is concentrated now in the areas of the kinks. It is interesting to notice that small and moderate thermal fluctuations facilitate the kink formation while for sufficiently high magnitudes of the noise intensity filaments takes on almost circular shape and the charge distribution is more uniform along the filament [see Figs. 9(d) and 10(d)].

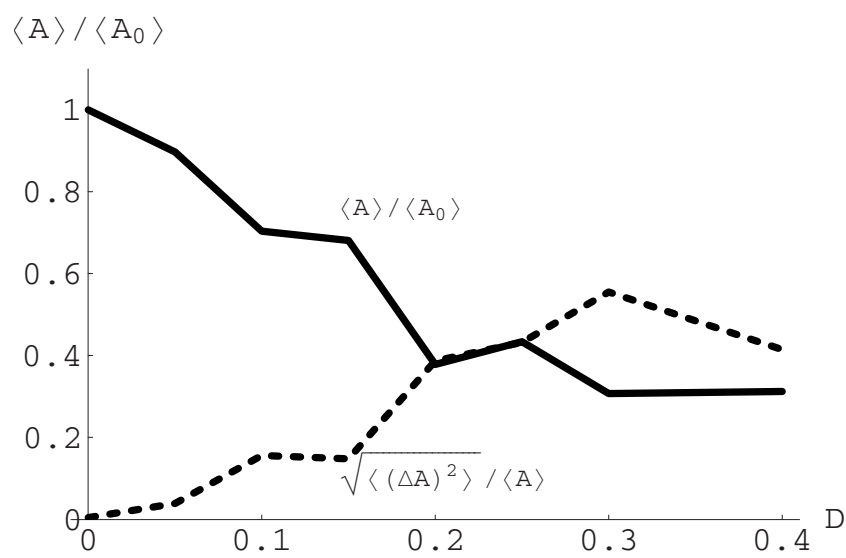

FIG. 8. Normalized mean value of the aspherity $\langle A\rangle /\left\langle A_{0}\right\rangle$ (solid line) and the relative standard deviation $\sqrt{\left\langle(\Delta A)^{2}\right\rangle} /\langle A\rangle$ (dashed line) versus noise for the case of softening with $\nu=0.29, \chi=2, \sigma=10^{5}$, and $J=0.22$.
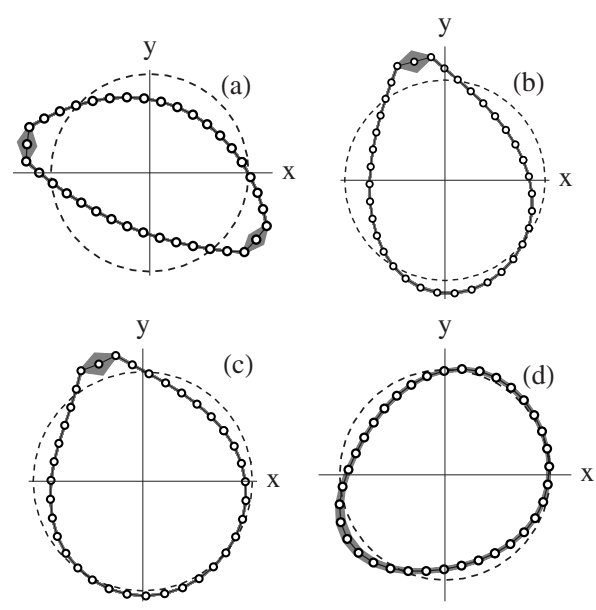

FIG. 9. Equilibrium configurations of the chain in the case of softening, $\nu=0.29, \chi=2, \sigma=10^{5}$, and $J=0.22$, for noise intensities $D=$ (a) 0.05 , (b) 0.10 , (c) 0.20 , and (d) 0.30 (solid line) against the initial circular conformation (dashed line). The gray shading represents the charge density.

We carried out a few runs of numerical simulations for a set of parameters that satisfy the inequality (60). We found out that for small enough $V$ the filament shape behavior and aspherity behavior are essentially the same as in the case of no interaction between charges. More detailed studies of effects of charge-charge interactions will be presented elsewhere.

\section{CONCLUSIONS AND DISCUSSION}

In this paper, we have investigated the role of thermal fluctuations on the charge-induced conformational transformations of closed semiflexible molecular chains. We have found that the results obtained in the mean-field approach [14] are rather robust in systems where the presence of charge hardens the local chain stiffness, the charge-curvature interaction counteracts the collapse of the chain and the mean-field picture survives. In the presence of white noise when the charge density and/or the strength of the chargecurvature coupling exceed a threshold value, the spatially uniform distribution of the charge along the chain and the circular, cylindrically symmetric shape of the chain become unstable. In this case the equilibrium state of the system is characterized by a spatially nonuniform charge distribution along the chain which takes on an ellipselike form. The transition to an anisotropic spatially nonuniform conformation is analogous to the phase transition of the second kind in the condensed matter physics.

In the case of hardening charge-bending interaction the charge and curvature distribution along the filament are smooth while for softening charge-bending interaction there are spontaneously created kinks where the smooth filament structure is disrupted. Almost the total amount of excess charge is concentrated in the vicinity of the kinks.

\section{ACKNOWLEDGMENTS}

Yu.B.G. and C.G. thank the Department of Mathematics, 

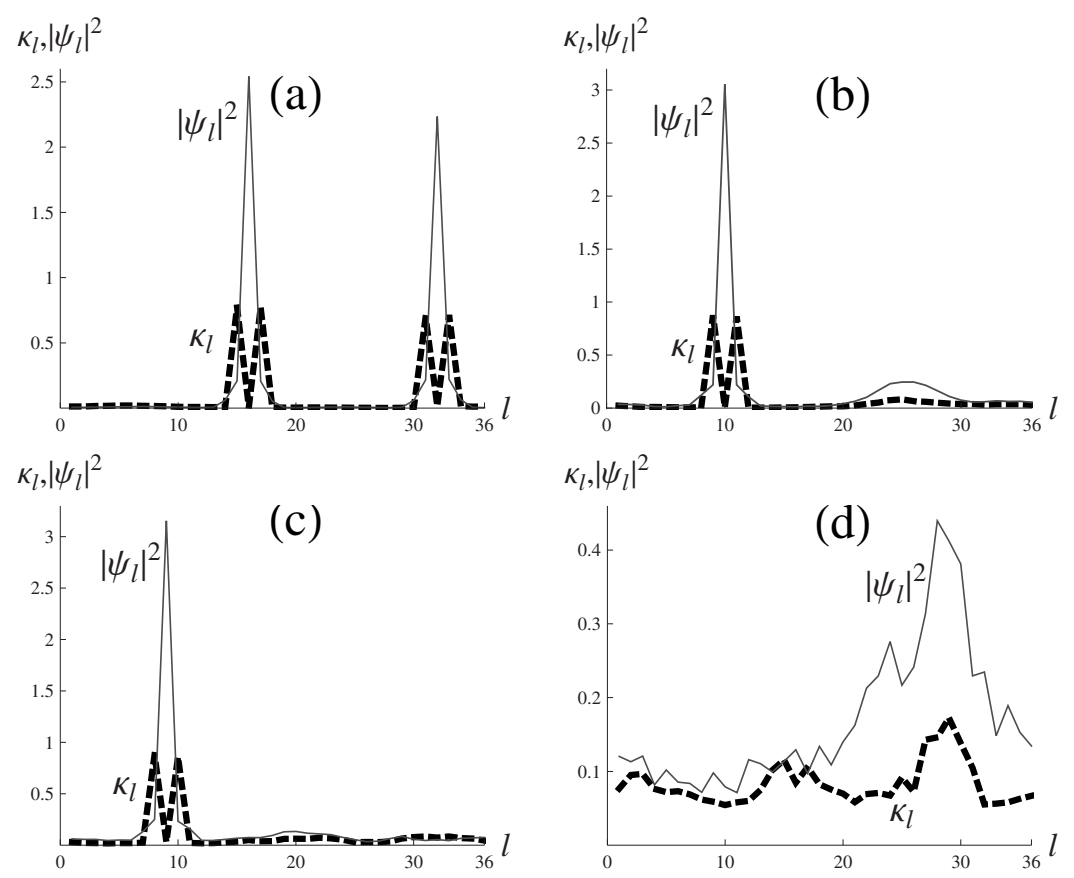

Technical University of Denmark, for hospitality. C.G. acknowledges Project No. MTM2007-62186 granted by the Spanish Ministerio de Educación y Ciencia. Yu.B.G. acknowledges also support from the Special Program of the Department of Physics and Astronomy of the National Academy of Sciences of Ukraine and Civilingeniør Frederik Christiansens Almennyttige Fond. M.P.S. acknowledges support from the European Union through the Network of Excellence BioSim, Grant No. LSHB-CT-2004-005137. We ac- knowledge financial support from the Danish Center for Applied Mathematics and Mechanics (DCAMM), the International Graduate Research School Contract No. 646-06004, and the Danish Agency for Science, Technology and Innovation. Finally, this work received funding from the Mathematical Network in Modelling, Estimation and Control of Biotechnological Systems (MECOBS), Contract No. 27405-0589, of the Danish Research Agency for Technology and Production.
[1] S. Hyde, A. Anderson, K. Larsson, Z. Blum, T. Landh, S. Lidin, and B. W. Ninham, The Language of Shape, 3rd ed. (Elsevier, Amsterdam, 1997).

[2] A. L. Kholodenko and T. A. Vilgis, Phys. Rep. 298, 254 (1998).

[3] M. Doi and S. F. Edwards, The Theory of Polymer Dynamics (Clarendon Press, Oxford, 1986).

[4] J. Wolfgang, S. M. Risser, S. Priyardshy, and D. N. Beratan, J. Phys. Chem. 101, 2986 (1997).

[5] J. Feitelson and G. McLendon, Biochemistry 30, 5051 (1991).

[6] T. J. Richmond and C. A. Davey, Nature (London) 423, 145 (2003).

[7] T. E. Cloutier and J. Widom, Mol. Cell 14, 355 (2004).

[8] Q. Du, C. Smith, N. Shiffeldrim, M. Vologodskaia, and A. Vologodskii, Proc. Natl. Acad. Sci. U.S.A. 102, 5397 (2005).

[9] C. Yuan, H. Chen, X. W. Lou, and L. A. Archer, Phys. Rev. Lett. 100, 018102 (2008).

[10] J. Yan and J. F. Marko, Phys. Rev. Lett. 93, 108108 (2004).

[11] P. A. Wiggins, R. Phillips, and P. C. Nelson, Phys. Rev. E 71,
021909 (2005).

[12] Q. Du, A. Kotlyar, and A. Vologodskii, Nucleic Acids Res. 36, 1120 (2007).

[13] P. A. Wiggins and P. C. Nelson, Phys. Rev. E 73, 031906 (2006).

[14] Y. B. Gaididei, P. L. Christiansen, and W. J. Zakrzewski, Phys. Rev. E 74, 021914 (2006).

[15] K. Solc, J. Chem. Phys. 55, 335 (1971).

[16] J. Rudnick and G. Gaspari, J. Phys. A 19, L191 (1986).

[17] C. W. Gardiner, Handbook of Stochastic Methods: For Physics, Chemistry and the Natural Sciences, 2nd ed. (Spinger, Berlin, 1996).

[18] Handbook of Mathematical Functions, edited by M. Abramowitz and I. Stegun (Dover Publications, New York, 1972).

[19] P. E. Kloeden and E. Platen, Numerical Solution of Stochastic Differential Equations, 3rd ed. (Springer, Berlin, 2004).

[20] B. W. Brown, J. Lovato, K. Russell, J. More, B. Garbow, and K. Hillstrom. 